\title{
Computer assisted selection and assessment of antibodies in the diagnosis of lymphomas
}

\author{
P J R Harkin, S A Kelly, A S Jack
}

\begin{abstract}
With increasing numbers of reagents the problem of selecting appropriate antibodies to solve problems in the diagnosis of lymphoma is becoming more complex. One approach is to use a computer program to optimise the selection process. Such a program was devised, incorporating data from an extensive literature search. When presented with a differential diagnosis it selects the most appropriate antibody panel and when given the results evaluates the relative likelihood of each possible diagnosis. In a retrospective study $81 \%$ of the tests used had been non-discriminatory, but using the results of the remaining $19 \%$ of the tests, the computer was able to select the "correct" diagnosis with a high degree of certainty. The development and use of this system illustrated several problems in the application of computer assisted diagnostic techniques in histopathology. These problems include incomplete data and lack of understanding of the process of histopathological diagnosis.
\end{abstract}

Computer assisted diagnostic systems have been developed in a variety of areas of medicine. ${ }^{1-3}$ Applications include the assessment of diagnostic probabilities in patients with acute abdominal pain ${ }^{4-6}$ to more particular tasks such as the accurate calculation of drug doses. The use of such methods in histopathology is very rare although there are a few published examples such as a program to assist in the diagnosis of breast disease on fine needle aspiration cytology. ${ }^{7}$

A particular area of pathology which seemed to lend itself to a computer based approach was the use of immunocytochemistry in the diagnosis of malignant lymphomas. The reason for choosing this area was firstly that the number of reagents available is now enormous: over 70 antibody clusters have now been defined and there are numerous unclassified antibodies in use; secondly, there are over 40 diagnostic categories in current use. Various attempts have been made to rationalise the selection of antibodies, ${ }^{89}$ but despite this, lymphoid immunocytochemistry is becoming increasingly inaccessible to the nonspecialist. The aim of this study was to devise a computer program which assisted in the selection of antibody panels for particular cases and in doing so provided the maximum useful information combined with economy of expensive reagents.

\section{Methods}

A literature search was performed to obtain data on the immunophenotype of malignant lymphomas (tables 1 and 2). All usable information from the Journal of Pathology, the Journal of Clinical Pathology, Human Pathology, the American Journal of Pathology, the American Journal of Clinical Pathology, the American Journal of Surgical Pathology, Cancer and Histopathology for the years 1984 to 1989 was collated using SuperCalc (Computer Associates International Inc, New York), a "spread sheet" program running on an IBM PS $/ 2$ computer. The data were manipulated and searched using software written by one of us (PJRH) in Turbo Pascal (Borland International Inc, Scotts Valley, California). Information was categorised as "usable" if tumours were discussed in terms of the proportion of cases positive or negative with particular reagents.

Table 1 Tumours considered in the study

\begin{tabular}{ll}
\hline B cell: & Low grade \\
& Lymphocytic \\
& Lymphoplasmacytoid \\
& Plasma cell myeloma \\
& Centrocytic \\
& Centrocytic/centroblastic (all types) \\
& High grade \\
& Lymphoblastic \\
& Centroblastic \\
& Immunoblastic \\
& Lowgrade \\
& Lymphocytic \\
Terebriform and mycosis fungoides \\
& AIL like lymphoma \\
& Lennerts'lymphoma \\
& Tzone lymphoma \\
& Pleomorphic small cell \\
& Peripheral T cell tumour \\
& High grade: \\
& Lymphoblastic \\
& Pleomorphic medium/large cell \\
& Immunoblastic \\
& Nodular sclerosing \\
& Lymphocyte predominant-nodular \\
& Lymphocyte predominant-diffuse \\
Hixed cellularity & Lymphocyte depleted \\
Hodgkin's disease: & K1 anaplastic \\
& Mediastinal B cell tumour \\
& Hairy cell leukaemia \\
&
\end{tabular}

Table 2 Antibodies used in study (listed by CD numbers where applicable)

IgG, IgM, IgD and IgA

$\kappa$ and $\lambda$

CD1 2, 3, 4, 5, 7, 8, 9, 10, 11a-c, 15, 19, 20, 21, 22, 23, 24, 25, $30,38,45$ and $45 \mathrm{r}$

leu6, leu7, leu8 and leu15

HLA-DR, EMA, tdt, C3br, MT1, MT2, MB1, MB2,

UCHL1, L26, L27, Mac, To5, Bf1, lysozyme, $\alpha-1$-anti-trypsin and $\mathrm{Ca} 2$ 
The computer was asked to perform two assessments of the data. Firstly, it was required to select the "best" antibodies for distinguishing between the two most likely differential diagnoses, using a discrimination algorithm, and secondly, to evaluate the results of using a panel of antibodies. This assessment was performed using a modified form of Bayes' algorithm.

The Discrimination Index (DI) is an estimate of the usefulness of a test (in our study antibody staining) in discriminating between two conditions. It is calculated using a weighted geometric mean of the probabilities of positive and negative staining for each condition. It is a real number in the range 1 (no value) to infinity (perfectly discriminant).

The pathologist making the initial histological assessment of the case subjectively assigned a relative probability to each differential diagnosis. In the absence of this information the computer calculated the prior probability of each diagnosis using local incidence data for each tumour type. This information was obtained from over 5000 cases on file. This prior probability estimate was used in conjunction with the results of immunocytochemistry to calculate the final odds ratio of the differential diagnosis. This calculation was performed using Bayes' algorithm.

Bayes' algorithm has been used in many computer assisted decision making systems. ${ }^{10}$ In essence it allows the combination of many individual test results to give a final odds ratio. To use the algorithm for a given test and diagnostic category it is necessary to know the following: $D_{i}$, the odds ratio for the initial prevalence of the disease; $\left(\mathbf{P}_{+} \mid \mathrm{D}_{+}\right)$and $\left(\mathbf{P}_{-} \mid \mathrm{D}_{+}\right)$, which are, respectively, the probabilities of a positive or negative test result, given that the disease is present; and $\left(\mathbf{P}_{+} \mid \mathbf{D}_{-}\right)$, and $\left(\mathbf{P}_{-} \mid \mathbf{D}_{-}\right)$, which are the equivalent probabilities, given that the disease is not present. From these it is possible to calculate the $D_{p}$, post-test probability from equation 1 for a positive test result or from equation 2 for a negative result.

$$
\begin{array}{r}
D_{p}=D_{i} \times \frac{\left(P_{+} \mid D_{+}\right)}{\left(P_{+} \mid D_{-}\right)} \\
\text {Equation 1 }
\end{array}
$$

$$
D_{p}=D_{i} \times \frac{\left(P_{-} \mid D_{+}\right)}{\left(P_{-} \mid D_{-}\right)}
$$

Equation 2

The value $D_{p}$ becomes $D_{i}$ for the assessment of the next test, and the process is continued until all tests have been assessed. Finally, the odds ratios are converted back to probabilities. Although the principle is simple, implementing Bayes' theorem requires much tedious calculation. Our study required over 2000 iterations per case. This is an ideal task for a computer.

\section{Results}

DATABASE CONSTRUCTION

The literature search for this work was comprehensive. Despite this the database has several gaps-antibody/diagnosis combinations for which no information has been published. Secondly, it was very rare for authors to state the reliability of antibodies as a proportion of cases tested in which the result was poor or uninterpretable.

\section{RESULTS OF RETROSPECTIVE STUDY}

In the cases studied a total of 395 immunocytochemical tests were performed. On the basis of the major pathological differential diagnosis, the proportion which the system regarded as useful-that is, achieved an arbitrary DI of 5.0 or higher discriminate tests-was $19^{\circ}{ }_{0}(n=76)$. Using the prior probabilities assigned at the time of histological review and only the tests regarded as discriminate, the system produced complete concordance with the original reported diagnosis almost always with a very high odds ratio (table 3 ).

\section{Discussion}

Evaluating a system of this type is very difficult. Using our retrospective data it was clear that the existing method of standard panels was not providing much useful information in relation to the number of tests done. Furthermore, most differential diagnoses can be resolved with a small number of tests. A prospective evaluation was carried out but the pathologist involved had also participated in the design of the system and had access to the tabulated data. It is therefore difficult to decide what contribution to day to day working the computer actually made. The impact is likely to be greater in centres handling only a few cases. To a large extent, however, the judgment is a purely subjective one as to whether an individual finds the system useful in his daily work.

The weakness of our system is the data input. Firstly, the data set was incomplete. Published data are selected by journals and by investigators for interest: it is incomplete to perhaps a surprising degree. Secondly, the reliability of a test is a vital component. A test may be very specific but give a technically satisfactory result in only a small number of cases. The number of times this is referred to in published work is exceptionally small and yet if a test is to be applied routinely this is critical information, particularly if the unsatisfactory result is due to fixation or processing and can be misinterpreted. Perhaps editors should require such information.

To use Bayes' theorem the data set should be complete and systematically collected for the population to which the calculation will be applied. In this type of application it is doubtful whether any centre has the resources to achieve this, given the number of diagnostic categories and the number of antibodies.

The method which was used to calculate the DI was one of several considered. These were 
Table 3 Numbers of tests performed on cases in retrospective study

\begin{tabular}{lllll}
\hline $\begin{array}{l}\text { No of tests } \\
\text { performed initially }\end{array}$ & $\begin{array}{l}\text { No of tests } \\
\text { assessed as useful } \\
\text { by computer }\end{array}$ & Overlap & $\begin{array}{l}\text { No of tests } \\
\text { used }\end{array}$ & $\begin{array}{l}\text { Final odds } \\
\text { ratio }\end{array}$ \\
\hline 9 & 3 & 0 & 3 & $1 \cdot 47$ \\
11 & 7 & 1 & 1 & $8 \cdot 6$ \\
19 & 1 & 0 & 3 & $14 \cdot 4$ \\
9 & 0 & 0 & 1 & $19 \cdot 6$ \\
17 & 11 & 3 & 4 & $96 \cdot 4$ \\
21 & 1 & 0 & 3 & 146 \\
16 & 2 & 0 & 3 & 160 \\
15 & 10 & 3 & 2 & 264 \\
21 & 7 & 3 & 3 & 1250 \\
15 & 13 & 1 & 1 & 3730 \\
18 & 14 & 4 & 4 & 5680 \\
14 & 7 & 2 & 2 & 12880 \\
13 & 13 & 2 & 2 & 16300 \\
10 & 14 & 4 & 3 & 24900 \\
16 & 10 & 4 & 4 & 40791 \\
16 & 13 & 1 & 2 & 52760 \\
14 & 1 & 2 & 5 & $7 \cdot 2 \times 10^{9}$ \\
25 & 20 & 6 & 4 & $7 \cdot 8 \times 10^{9}$ \\
15 & 19 & 6 & 3 & $1 \cdot 7 \times 10^{10}$ \\
18 & 17 & 7 & 3 & $2 \cdot 3 \times 10^{11}$ \\
22 & 22 & 7 & 5 & $6 \cdot 4 \times 10^{15}$ \\
19 & 11 & 8 & 3 & $8 \cdot 3 \times 10^{15}$ \\
16 & 17 & 2 & 3 & \\
10 & 11 & 6 & 4 & \\
16 & & 4 & 4 & \\
\hline
\end{tabular}

Table 4 Test result matrices

\begin{tabular}{lll}
\hline Test 1 & + & - \\
$\mathrm{D}_{1}$ & 90 & 10 \\
$\mathrm{D}_{2}$ & 10 & 90 \\
Test 2 & + & - \\
$\mathrm{D}_{1}$ & $99 \cdot 9$ & $0 \cdot 1$ \\
$\mathrm{D}_{2}$ & 90 & 10 \\
\hline
\end{tabular}

assessed on a number of test cases, both real and hypothetical, and the chosen method was clearly superior. Initially, to assign an objective value to the ability of an antibody to discriminate between two tumours, the computer assessed the relative predictive values of positive and negative staining of each tumour and calculated their geometric mean. This simple index was found to be unreliable when very small probabilities were involved, and so a weighted selection system was developed.

The calculated discrimination index (DI) is an attempt to reconcile the sensitivity and specificity of the test into a single measurement. This has many problems. Consider the two test result matrices illustrated in table 4 . Test 1 , if positive, gives a probability ratio of $90: 10$ in favour of diagnosis $D_{1}$. Similarly, a negative result gives a probability of 90:10 in favour of diagnosis $\mathrm{D}_{2}$. Using our initial method (equation 3 ) of calculating the

$$
D I=\sqrt{e^{\left|\ln \left(\frac{P_{+} \mid D_{+}}{P_{+} \mid D_{-}}\right)\right|+\left|\ln \left(\frac{P_{-} \mid D_{+}}{P_{-} \mid D_{-}}\right)\right|}}
$$

Equation 3

DI we obtain the value of $9 \cdot 0$. Now consider test 2 . Intuitively, this is a less suitable test as both tumours tend to stain positively, but the simple DI formula calculates out as 10.54. This is because a negative result (which is unlikely) would be extremely discriminant.

To overcome this problem, we introduced a weighting factor based on the likelihood of each result. This weights the probability ratio for each result in proportion to its probability

$$
\begin{array}{r}
D I=\sqrt{e^{\left|\ln \left(\boldsymbol{P}_{+} \times \frac{\boldsymbol{P}_{+} \mid D_{+}}{\boldsymbol{P}_{+} \mid D_{-}}\right)\right|+\left|\ln \left(\boldsymbol{P}_{-} \times \frac{\boldsymbol{P}_{-} \mid D_{+}}{\boldsymbol{P}_{-} \mid D_{-}}\right)\right|}} \\
\text {Equation 4 }
\end{array}
$$

of occurring. With this new formula (equation 4 ), the DI for test 1 is still $9 \cdot 0$, but the DI for test 2 is now $4 \cdot 61$. The major disadvantage of this method is that it places a much greater emphasis on the accuracy of the pre-test probability values assigned to each condition. We used estimates of pre-test probability provided by the assessing pathologist (ASJ) and derived from local incidence data taken from departmental records for different sections of the study.

A further pathological limitation is worthy of note. The distinction of benign from malignant cannot be made by this method. If immunocytochemistry has a role in this area at all it is in the detection of aberrant phenotypes. ${ }^{11-13}$ The data required to implement this are relatively small: in computer terms this would require a rule based, rather than a probability based, system.

A use of this type of system at a more general level than the individual case is to test the diagnostic value of immunocytochemistry in particular diagnostic problems. One such is the distinction of various types of $\mathrm{T}$ cell lymphoma from Hodgkin's disease. The literature in this area is extensive and using the data our system found that one or any combination of antibodies is ineffective at solving this problem of differential diagnosis. This statement ignores subtle differences in patterns of staining, which may be important. ${ }^{14}$ Again, this is not systematically recorded in published literature.

Could this type of approach be applied in other areas of histopathology? To answer this question requires knowledge of how pathologists work. It could be argued that the pathologist uses a mental questionnaire with a series of yes or no answers or that observations are made and the diagnosis formulated. Although there are no formal data in this area, neither is likely to be true. The trained individual may very quickly assess a section of tissue, list a small number of possible diagnoses, and then seek discriminatory evidence. In theory a computer would be best used in guiding the pathologist towards the evidence to look for to test his diagnoses. This could be based on probabilities as in the present study or on a series of absolute rules based on definitions of disease process-for example, if feature $x$ is not present the diagnosis $\mathrm{A}$ is untenable. Although it is doubtful if such a system is of any help for the expert, such a system would be of great benefit as a teaching aid, particularly when combined with video disk based picture libraries. The problem in designing a system is not one of technology but of data. The information does not exist in usable form, not to mention disagreement among experts as to the importance of findings.

The work reported in this study is far from 
definitive. The system is effective in carrying out its designed task. Whether it is valuable is to an extent subjective but the experience of carrying out this work serves to illustrate the more general problems of computer applications of this type in pathology. It also highlights the question as to the process of pathological diagnosis which is worthy of further study.

1 De Bernadinis $M$, Violi V, Montanari M, Roncoroin L, Peracchia A. Early detection of necrotising pancreatitis by computer. Ital J Surg Sci 1986;16:269-74.

2 McIntyre N. Computer-assisted diagnosis in jaundice and liver disease. J Hepatol 1989;3:269-72.

3 Edwards FH, Schaefer PS, Callahan S, Graeber GM, Albus RA. Bayesian statistical theory in the preoperative diagnosis of pulmonary lesions. Chest 1987;95:888-91.

4 Burton ID. Acute appendicitis in childhood: a feasibility study of computer assisted diagnosis. Aust NZ Surg study of compur

5 Kirkeby J, Rism C. Use of a computer system for diagnosing acute abdominal pain in a small hospital. Scand J Gas troenterol 1987;128(Suppl): 174-6.

6 Feyno G, Clamp SE, de Dombal FT, Wilczek H. Computer- aided diagnosis of 223 acute abdominal pain cases at Nacka Hospital Sweden. Scand J Gastroenterol 1987;128(Suppl): 178-9.

7 Heathfield HA, Kirkham N, Ellis IO, Winstanley G. Computer assisted diagnosis of the needle aspirate of the breast. $J$ Clin Pathol 1990;43:168-70.

8 Norton AJ, Isaacson PG. Invited review: Lymphoma phenotyping in formalin-fixed and paraffin wax-embedded tissue. Histopathol 1989;14:437-46.

9 Wissue. Histopathol 1989,14:437-46.

West KP, Warford A, Fray L, Allen M, Campbell AC, Lauder I. The demonstration of B-cell, T-cell and myeloid antigens in paraffin sections. J Pathol 1986; 150:89-101.

10 Edwards FH, Graeber GM. The theorem of Bayes as a clinical research tool. Surg Gynaecol Obstet 1987;165: 127-9.

11 Knowles DM. Immunophenotypic and antigen receptor gene rearrangement analysis in T-cell neoplasia. $A m J$ Pathol 1989;134:761-85.

12 Picker LJ, Weiss LM, Medeiros LJ, et al. Immunophenotypic criteria for the diagnosis of non-Hodgkins phenotypic criteria for the diagnosis of non
lymphoma. Am J Pathol 1987;128:181-201.

13 Hastrup N, Ralkiaer E, Pallesen G. Aberrant phenotypes in peripheral T-cell lymphomas. J Clin Pathol 1989;42:
pastrup N, Ralkiaer E, Pallesen G. Aberrant phenotypes in 398-402.

14 Pinkus GS, Premi T, Said J. Leu-M1-A marker for ReedSternberg cells in Hodgkins disease. An immunoperoxidase study of paraffin embedded tissues. Am J Pathol 1985;119:244-52. 